\title{
Neurectomia do nervo interósseo anterior e posterior para controle da dor de pacientes com artrose de punho
}

\section{Anterior and posterior interosseous nerve neurectomy for pain control in patients with arthrosis of the wrist joint}

\author{
João Marcos Lopes Moreira ${ }^{1}$. Marcos Levy Machado Barbalho Viana ${ }^{1}$. Yuri de Moraes Facó ${ }^{1}$ Luanna de \\ Queiroz Lemos². Gabriela Freire Bezerra3 ${ }^{3}$. Maria Luzete Costa Cavalcante ${ }^{4}$. \\ 1 Médico, Residente do Programa de Ortopedia/Traumatologia, Universidade Federal do Ceará (UFC), Fortaleza, Ceará, \\ Brasil. 2 Estudante de Medicina, Universidade Federal do Ceará (UFC), Fortaleza, Ceará, Brasil. 3 Mestranda no Programa de \\ Pós-graduação em Farmacologia, Universidade Federal do Ceará (UFC), Fortaleza, Ceará, Brasil. 4 Doutora em Ortopedia e \\ Traumatologia, Universidade de São Paulo (USP). Professora do Departamento de Cirurgia, Universidade Federal do Ceará (UFC), \\ Médica preceptora da Residência de Ortopedia/Traumatologia, Universidade Federal do Ceará (UFC), Fortaleza, Ceará, Brasil.
}

\section{RESUMO}

Introdução: a osteoartrose é a doença articular de maior ocorrência no mundo, sendo definida como uma insuficiência da cartilagem articular devido a fatores mecânicos, genéticos, hormonais, ósseos e metabólicos, acarretando um desequilíbrio entre degradação e síntese da cartilagem articular e do osso subcondral. Objetivos: este estudo avalia quantitativamente a dor dos pacientes selecionados para o procedimento de neurectomia. Material e métodos: estudo longitudinal, prospectivo e analítico, com abordagem quantitativa das variáveis através da avaliação e acompanhamento de pacientes com dor crônica no punho devido a artrose de tal articulação. Resultados: 55,6\% dos pacientes analisados eram do sexo masculino com variadas manifestações clínicas de dor e melhora do quadro álgico após o teste da xilocaína nos tempos de 5 e 10 minutos. A avaliação após 1 mês da neurectomia do nervo interósseo apresentou uma melhora significativa do quadro álgico, onde todos os pacientes analisados qualificaram a dor como fraca. Após 3 meses da cirurgia observou-se resultados semelhantes ao encontrado na avaliação após 1 mês da cirurgia; dos nove pacientes avaliados, apenas um relatou dor moderada. Conclusão: pacientes que foram submetidos a neurectomia do nervo interósseo apresentaram uma melhora significativa do quadro álgico associado a osteoartrose.

Palavras-chave: Denervação. Artrose. Articulação do punho.

\section{ABSTRACT}

Introduction: Osteoarthritis is the most common joint disease in the world. It is defined as the insufficiency of the articular cartilage due to mechanical, genetic, hormonal, bone and metabolic factors, which causes an imbalance between the degradation and synthesis of articular cartilage and the subchondral bone. Objectives: This study aims to evaluate quantitatively the pain level in the patients selected for neurectomy procedure. Methods: Longitudinal, prospective and analytical study. Quantitative approach of the variables by evaluation and monitoring of patients with chronic pain resulted of wrist joint arthrosis. Results: The majority of the patients analyzed, $55.6 \%$, were male and $44 \%$ female, who presented varied clinical manifestations of pain and consequent improvement after the xylocaine test at 5 and 10 minutes. The assessment after 1 month of interosseous nerve neurectomy showed a significant pain improvement, where all patients analyzed described it as weak pain. The evaluation after 3 months of surgery showed a similar result to that found in the evaluation after 1 month of surgery, among the 9 patients evaluated, only 1 reported moderate pain. Conclusion: Patients who were submitted to interosseous nerve neurectomy procedure showed a significant improvement in the pain associated to the osteoarthrosis condition.

Keywords: Denervation. Joint diseases. Wriste joint.

Autor correspondente: João Marcos Lopes Moreira, Rua Osvaldo Cruz, 188, Meireles, Fortaleza, Ceará. CEP: 60125-150. Telefone: +55 85 99968-5340. E-mail: joao_marcoslm@hotmail.com

Conflito de interesses: Não há qualquer conflito de interesses por parte de qualquer um dos autores.

Recebido em: 01 Set 2017; Revisado em: 14 Mar 2018; Aceito em: 14 Mar 2018. 


\section{INTRODUÇÃO}

A osteoartrose é definida como uma insuficiência da cartilagem articular decorrente de fatores mecânicos, genéticos, hormonais, ósseos e metabólicos, que acarretam um desequilíbrio entre a degradação e a síntese da cartilagem articular e do osso subcondral. A osteoartrose manifesta-se por alterações morfológicas, bioquímicas, moleculares e biomecânicas das células e da matriz extracelular que levam ao amolecimento, fibrilação, ulceração e perda da cartilagem articular, esclerose do osso subcondral, formação de osteófitos e cistos subcondrais. ${ }^{1}$

A osteoartrose é a maior causa de incapacidade na maioria das populações, estima-se que $80 \%$ dos indivíduos acometidos apresentam limitações de movimento. ${ }^{2}$ Um estudo realizado pela Organização Mundial da Saúde (OMS) refere que a osteoartrose seria a quarta causa mais importante de incapacidade entre mulheres e a oitava em homens. ${ }^{3}$

Ao analisar a osteoartrose pós-traumática do punho, a literatura demonstra que sua etiologia é, na maioria das vezes, secundária a lesões ligamentares, ou decorrente de fraturas do carpo. $^{4,5}$

A desnervação do punho é um novo procedimento com poucas publicações na literatura, mas que tem sido utilizada cada vez mais pelos cirurgiões de mão nos punhos dolorosos com artrose, especialmente na Europa Central. ${ }^{6}$ A desnervação por neurectomia articular se define como um procedimento cirúrgico de interrupção da condução da dor de uma articulação ao sistema nervoso central, pela secção dos ramos nervosos articulares. O objetivo é suprimir ou diminuir a dor, mas mantendo a mobilidade articular, sem afetar também a sensibilidade cutânea. ${ }^{7}$ De tal forma, muitos indivíduos poderão prolongar ainda mais a necessidade da cirurgia mais complexa, como a artrodese, mas ainda com o bônus de melhora da qualidade de vida.

Esse estudo teve como propósito avaliar quantitativamente a dor dos pacientes selecionados para o procedimento de neurectomia. Foi feita uma avaliação da dor antes do procedimento e após a realização do mesmo, na busca de resultados estatisticamente relevantes que respaldem e consolidem essa técnica como uma opção mais rápida e satisfatória na recuperação da funcionalidade dos acometidos.

\section{MATERIAL E MÉTODO}

Trata-se de um estudo longitudinal, prospectivo e analítico, com abordagem quantitativa das variáveis através da avaliação e acompanhamento de pacientes com dor crônica no punho em decorrência de artrose das articulações do punho. $\mathrm{O}$ estudo foi realizado no ambulatório especializado em mão e punho do Hospital Universitário Walter Cantídio (HUWC) em Fortaleza, Ceará.

Foram eleitos para participar do estudo pacientes de ambos os sexos com artrose de punho acompanhados rotineiramente no serviço de Ortopedia do HUWC no período de 2009 a
2016, que apresentaram sintoma álgico crônico refratário às alternativas clínicas com indicação terapêutica de artrodese de punho. A pesquisa ocorreu no período de janeiro de 2016 a julho de 2017, sendo excluídos os pacientes diagnosticados com artrose do punho assintomática, indivíduos diagnosticados com patologias imunomediadas, doenças reumatológicas ou ainda os indivíduos que não tenham indicação de artrodese de punho.

Os dados foram coletados por meio de questionário criado para o desenvolvimento desta pesquisa, sendo aplicado para realização de triagem dos pacientes antes dos mesmos serem submetidos à neurectomia do nervo interósseo anterior e posterior do punho. Esse mesmo instrumento foi utilizado para acompanhar de forma quantitativa o sintoma álgico dos pacientes nos meses seguintes à realização do procedimento cirúrgico. O questionário reuniu informações sobre a identificação do paciente, condição patológica de base para artrose de punho, dados sobre limitação funcional articular e graduação do sintoma doloroso por meio de escala numérica e facial da dor.

Por meio do questionário, foi registrado também o resultado do teste da xilocaína, procedimento que possibilita análise quantitativa subjetiva da dor pelo próprio paciente. Aplica-se xilocaína $(5 \mathrm{ml})$, anestésico local, para o bloqueio do nervo interósseo anterior e posterior do antebraço. Após 1 minuto do procedimento, é solicitado ao paciente que novamente atribua classificações a sua dor, utilizando a escala numérica e a escala facial da dor. Repete-se a classificação do sintoma álgico no quinto e décimo minuto após o procedimento. Após aplicação do teste de xilocaína os pacientes foram selecionados para realização da neurectomia. Após realização do procedimento cirúrgico foram reavaliados e aplicados o questionário para quantificação da dor no pós-operatório com 1 mês e 3 meses da realização da cirurgia.

Após a etapa processamento manual, os dados foram alocados no Microsoft Office Excel 2003, com espaços segundo classificações pré-estabelecidas, para análise e interpretação. Foram realizadas análises descritivas e análises de frequências representadas em gráficos de histograma. Foi realizado Teste-T para amostras pareadas com o intuito de comparar as médias dos grupos e observar a significância. Foi considerado significativo $\mathrm{P}<0,05$. As exposições das informações processadas foram obtidas no programa SPSS versão 20 para MAC OSX e exposta em gráficos.

O estudo foi aprovado pelo Comitê de Ética da Universidade Federal do Ceará (UFC), respeitando os princípios éticos contidos na resolução 466/12 sobre pesquisa com seres humanos do Conselho Nacional de Saúde/Ministério da Saúde de 1996. CAAE: 57613916.4.0000.5045.

\section{RESULTADOS}

No período a que se refere o presente estudo foram analisados nove pacientes com artrose do punho que foram acompanhados 
no ambulatório de ortopedia do HUWC. Cinco pacientes eram do sexo masculino $(55,6 \%)$ e quatro do sexo feminino $(44,4 \%)$. Esses pacientes responderam o questionário com o objetivo de se obter quantitativamente o percentual de dor que cada um sentia. Destes, um paciente apresentou na escala de dor resultado máximo 10 (insuportável), caracterizada como dor contínua, que prejudica suas atividades laborais e referida pelo mesmo como a dor mais intensa de sua vida, enquanto seis pacientes obtiveram o resultado 7 (intensa) na escala de dor, caracterizadas como dor não contínua, que prejudica suas atividades e sendo a mais intensa da vida. Já para dois pacientes o resultado foi 5 (moderada), cuja dor é não contínua, prejudicam sua atividade laboral e não refere como a mais intensa de sua vida.

Houve melhora considerável do quadro álgico dos pacientes após realização do teste da xilocaína nos tempos 5 e 10 minutos respectivamente. Após 5 minutos da aplicação apenas um paciente apresentava dor 5 (moderada) e outros oito apresentaram dor 3 (dor fraca) da escala de dor aplicada neste presente estudo. Após 10 minutos o alívio da dor foi ainda maior com todos apresentando dor fraca e/ou ausência da dor. Três pacientes apresentaram dor 3 (fraca), dois deles apresentaram dor 2 (fraca) e um com dor 1 (fraca). Para outros três o alívio foi ainda maior referindo ausência da dor. Todos os pacientes que foram submetidos a realização do teste de xilocaína realizaram o procedimento cirúrgico de neurectomia do nervo interósseo anterior e posterior e afirmaram considerar importante a melhora parcial da dor após o teste da xilocaína.

Os pacientes foram avaliados após 1 mês da neurectomia do interósseo, e o resultado obtido mostrou a melhora da dor após o procedimento. Dois pacientes apresentaram dor 1 (fraca), seis apresentaram dor 2 (fraca) e apenas um paciente apresentou dor 3 (fraca).

Na avaliação após 3 meses da neurectomia do interósseo, observou-se resultado semelhante encontrado na avaliação após 1 mês da cirurgia. Dos nove pacientes, um relatou voltar a sentir dor marcada na escala como 5 (moderada), outro relatou dor 3 (fraca), quatro relataram sentir a dor 2 (fraca) e três relataram sentir a dor 1 (fraca). Esse resultado mostra que dos 9 pacientes apenas 1 voltou a sentir uma dor considerada moderada pela escala e todos os outros estavam com resultado semelhante a avaliação após 1 mês.

No Gráfico 1, análise do Teste $\mathrm{T}$ para amostras pareadas, observa-se a comparação das médias dos níveis de dor \pm desvio padrão, onde os resultados mostram uma diferença significativa entre as médias dos níveis de dor antes da cirurgia quando comparadas as médias dos testes de xilocaína e do procedimento cirúrgico de neurectomia do nervo interósseo. $\mathrm{P}<0,001$ : Pré-cirurgia vs todos os demais grupos. $\mathrm{P}<0,01$ : Pós xilocaína $5 \mathrm{~min}$ vs pós xilocaína 10 min e 1 mês após a cirurgia.

Gráfico 1. Comparativo das médias dos níveis de dor antes da cirurgia e após a cirurgia. HUWC-UFC, 2009-2016.

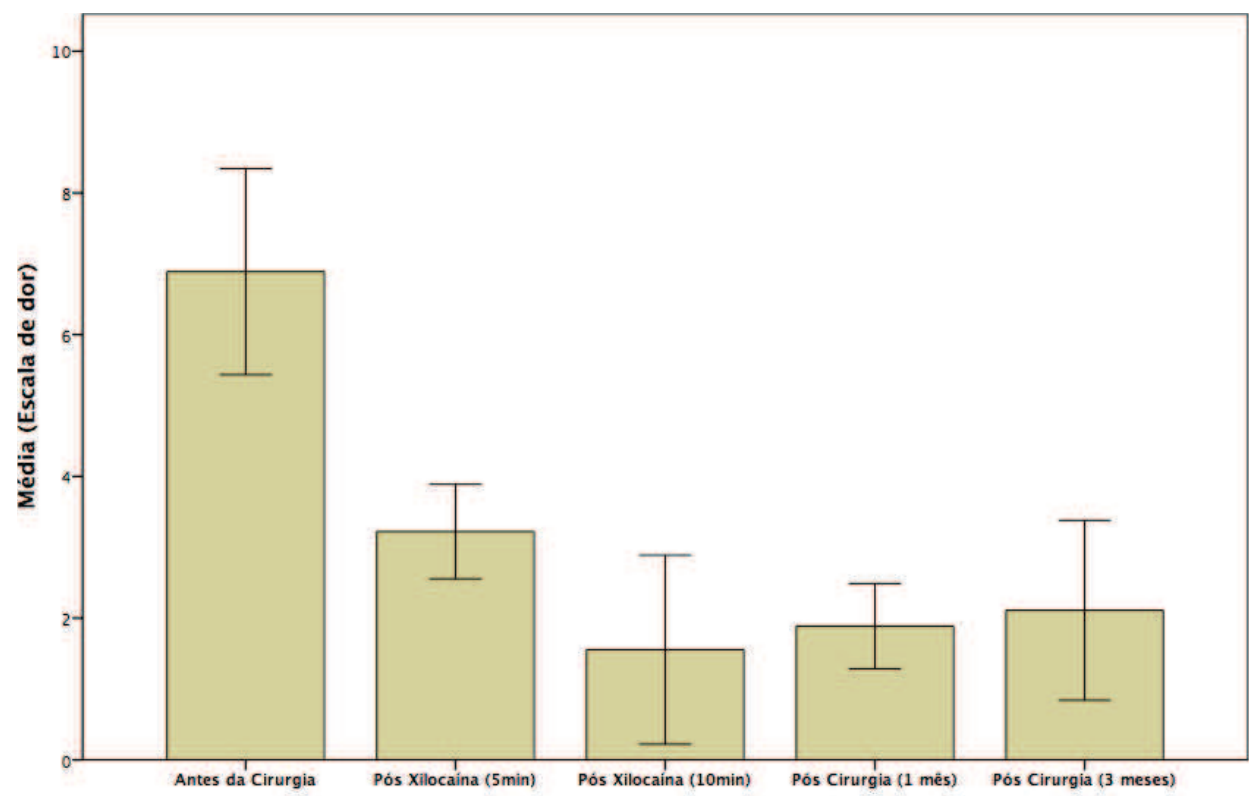

\section{DISCUSSÃO}

A osteoartrose é uma doença articular degenerativa, uma resposta complexa dos tecidos articulares à idade, fatores genéticos e ambientais, sendo caracterizada por uma degeneração da cartilagem, remodelamento e hipercrescimento ósseo e constituindo a principal causa de dor articular e incapacidade nos pacientes de meia idade e idosos. ${ }^{8-10}$
Ao analisar a osteoartrose pós-traumática do punho a literatura demonstra que sua etiologia é, na maioria das vezes, secundária a lesões ligamentares ou a fraturas do carpo. Estudos epidemiológicos mostram que $43,6 \%$ da incidência geral de traumas acometem o punho e a mão, dos quais $16,7 \%$ apresentariam envolvimento dos ossos do carpo. ${ }^{1,2}$

Uma consideração anatômica importante é com relação 
à inervação sensitiva do punho, que provem dos nervos interósseo anterior e cutâneo palmar (ramos do nervo mediano), ramo cutâneo dorsal do nervo ulnar, ramo superficial do nervo radial e nervo interósseo posterior (originados do nervo radial). Os nervos interósseo anterior e o posterior são responsáveis por $2 / 3$ da inervação da cápsula articular volar e dorsal, respectivamente. ${ }^{7,11}$

O padrão mais comum de artrite degenerativa do punho é o colapso avançado escafo-semilunar ou SLAC (scapholunate advanced collapse). A lesão corresponde a uma sequencia de mudanças degenerativas e progressivas secundárias ao desalinhamento entre o escafoide, semilunar e rádio, causado principalmente pela subluxação rotatória e/ou pseudartrose do escafoide. O SLAC secundário à pseudartrose do escafoide é melhor denominado SNAC (Scaphoid Nonunion Advanced Collapse). ${ }^{11-13}$

O sintoma mais importante e que traz o paciente ao médico é a dor localizada no sítio anatômico. A dor no início da doença costuma ocorrer com o uso da articulação, mas a evolução da doença faz a dor assumir caráter persistente mesmo no repouso e esteja associada a rigidez articular. ${ }^{8,14,15}$

Várias abordagens cirúrgicas são descritas para o tratamento da osteoartrose pós-traumática do carpo. Tradicionalmente, a artrodese do punho é o tratamento para as artrites do punho que não respondem ao tratamento com medicamentos anti-inflamatórios, imobilizadores e injeções com corticosteroides., ${ }^{2,16}$ Contudo, embora produza como resultado um punho indolor e estável, a artrodese do punho ainda é motivo de controvérsia no que se refere à função global do membro afetado. ${ }^{2,16,17}$

A carpectomia proximal e a artrodese dos quatro cantos são opções de tratamento que preservam certo grau de mobilidade do punho artrítico quando comparadas com a artrodese total. ${ }^{1,16}$ A carpectomia proximal é um procedimento tecnicamente mais simples, porém, leva ao encurtamento do carpo e forma uma nova articulação relativamente incongruente entre o capitato e a fossa semilunar do rádio. ${ }^{11,18,19}$ Enquanto a artrodese dos quatro cantos mantém intacta a articulação radiossemilunar, porém, é tecnicamente mais complexa, necessitando de um período maior de imobilização pós-operatória, além da possibilidade da não união dos ossos, da infecção do material de síntese e impacção dorsal radiocapitato. ${ }^{1,15,20}$

Ainda existe bastante controvérsia quanto ao procedimento de salvamento ideal para pacientes portadores de artrose do punho secundária ao SLAC. ${ }^{6}$ Nesse contexto, a desnervaçäo do punho é um novo procedimento com poucas publicações na literatura, mas que tem sido utilizado cada vez mais pelos cirurgiões de mão nos punhos dolorosos com artrose, porque muitas vezes é evitada artrodese e pode-se obter um punho livre de dor, com preservação da mobilidade., ${ }^{6,21,22}$

A desnervação para o tratamento da dor do punho evoluiu da desnervação total do punho à desnervação do nervo interósseo posterior e anterior. A desnervação do punho oferece a vantagem do alívio da dor, minimizando a imobilização prolongada, a perda de movimento e a atrofia muscular, muitas vezes associada a reconstruções cirúrgicas ou a artrodese limitada do carpo. A desnervação total do punho foi descrita por Wilhelm para a paliação da dor crônica do punho associada à artrose degenerativa, doença de Kienböck e afecções do carpo. Posteriormente ao relato de Wilhelm, a desnervação total do punho foi defendida para osteoartrite primária, artrose pós-traumática, fraturas do rádio distal intra-articular, doença de Kienböck e fraturas do carpo. ${ }^{22}$

Buck-Gramcko (1977), ${ }^{23}$ descreveu em seu estudo que $69 \%$ dos pacientes submetidos a desnervação do punho apresentaram uma melhora evidente do quadro álgico associado a artrose do punho. Estes se mostraram livres de dor ou relataram dor após atividades de alta intensidade. Nesse mesmo estudo, 26\% dos pacientes investigados apresentaram melhora da amplitude de movimento e redução do quadro álgico em comparação com o quadro clínico pré-operatório. De forma semelhante este estudo demonstrou, no pós-operatório, nos períodos de um e três meses melhora significativa do quadro doloroso.

No primeiro mês todos os pacientes investigados qualificaram a dor como do tipo fraca, já após três meses oito pacientes relataram dor fraca e um paciente ralatou dor moderada, o que se dá devido à dificuldade de desnervação completa do punho e à riqueza de anastomoses dos ramos nervosos articulares. ${ }^{6}$

Radu et $\mathrm{al}^{24}$ realizaram um trabalho longitudinal com pacientes submetidos a desnervação do punho, acompanhando os pacientes por um tempo médio de 51(18-97) meses. Em seu trabalho, o autor também realizou teste com anestésico local previamente ao procedimento cirúrgico em alguns de seus pacientes, a fim de compararar seus resultados com aqueles obtidos após a cirurgia. Seus pacientes foram divididos em três grupos de acordo com o diagnóstico de base. O grupo I era formado por paciente com artrose radiocarpal e instabilidade do carpo, o grupo II composto por indivíduos com artrose sem associação a instabilidade e o grupo III formado por paciente sem indicativos de artrose. Dos 26 pacientes submetidos ao teste com anestésico, dez relataram boa resposta, 10 tiveram resposta satisfatória e 6 tiveram resposta modesta. Dos 20 pacientes com resposta boa/satisfatória ao teste, apenas 13 (65\%) obtiveram melhora após a cirurgia. Em seu trabalho também foi relatado que $66 \%$ dos pacientes submetidos a denervação do punho se mantiveram sem dor a longo prazo. Nos grupos I, II e III o percentual de pacientes com melhora foi de, respectivamente, $64 \%, 63 \%$ e $85 \%$, mas apenas no grupo III esse percentual obteve significância estatística.

Braga-silva et $\mathrm{al}^{25}$ também demonstraram bons resultados com a desnervação do punho. Em seu trabalho, foram analisados 49 punhos desnervados com a técnica de Wilhelm. Dos pacientes, $68 \% \pm 8 \%$ obtiveram melhora no primeiro mês. O platô de melhora da dor foi obtido por volta de um ano, mantendo-se estável em $79 \% \pm 4 \%$ dos pacientes após 36 meses. Também foram registradas melhoras na amplitude de movimento em todos os eixos e um ganho de $23 \%$ de força na pegada. $\mathrm{O}$ estudo também mostrou que os pacientes se mantiveram com controle da dor em longo prazo, apesar da piora radiológica. 
Storey et $\mathrm{al}^{26}$ realizaram o seguimento de 37 pacientes submetidos a desnervação após boa resposta no teste com anestésico local. Na avaliação após 18 meses, os índices de dor tiveram queda de $60 \%$ quando comparados aos prévios à cirurgia. 30 desses pacientes ainda relataram melhora progressiva da dor.

Em seu trabalho Dellon et $\mathrm{al}^{27}$ mostraram resultados ainda mais positivos, obtendo melhora da dor em mais de $90 \%$ dos pacientes apenas com desnervação do interósseo posterior. Além disso, $83 \%$ de seus pacientes voltaram a trabalhar após o tratamento. Esses dados são compatíveis com aqueles obtidos por Weinstein ${ }^{28}$ em seu trabalho com 19 pacientes submetidos à desnervação dos interósseos anterior e posterior, que mostrou melhora da dor em $80 \%$ dos pacientes e melhora da força na pegada em $45 \%$ dos casos em sua avaliação após 2,5 anos da cirurgia. Além disso, $73 \%$ desses pacientes retornaram ao trabalho.

Um estudo publicado por Riches ${ }^{29}$ comparou o resultado de desnervação do interósseo posterior em 13 pacientes com os obtidos por artrodese do punho em 15 pacientes. Os pacientes foram reavaliados numa média de 39 meses e 22 meses, respectivamente, após artrodese e desnervação. O grupo submetido a artrodese teve melhora significativa da dor em $54 \%$ e dos escores funcionais em $36 \%$ dos casos durante o

\section{REFERÊNCIAS}

1. Severo LA, Lopes OV Júnior, Piluski P, Lech O. Análise funcional da artrodese dos quatro cantos comparada com a carpectomia proximal. Rev Bras Ortop. 2006;41(1/2):14-21.

2. Barbieri CH, Mazzer N, Elui VM, Fonseca MC. Resultados funcionais da artrodese do punho. Acta Ortop Bras. 2002;10(1):17-24.

3. Souza JM, Pardini AG Junior. Clínica ortopédica: atualização em osteoartroses. Rio de Janeiro: Guanabara Koogan; 2005.

4.Watson HK, Ruy J. Evolution of the arthritis of the wrist. Clin Orthop. 1986;202:57-67.

5. Watson HK, Ruy J, Akelman E. Limited triscaphoid intercarpal arthrodesis for rotatory subluxation of the scaphoid. J Bone and Join Surg. 1986;68:345-9.

6. Freitas AD, Pardini A Júnior, Tavares KE, Aguilar, RM. Denervaçäo do punho. Rev Bras Ortop. 1993;28(4):176-8.

7. Chambriaed C, Vasconcelos R, Couto P, Osório L, Menegassi Z. Artrodese do punho com placa de compressäo dinâmica. Rev Bras Ortop. 1998;33(5):409-12.

8. Coimbra IB, Pastor EH, Greve JM, Puccinelli ML, Fuller R, Cavalcanti FS, et al. Osteoartrite (artrose): tratamento. Rev Bras Reumatol. 2004;44(6):450-3.

9. Camanho GL, Imamura M, Arendt-nielsen L. Gênese da dor na artrose. Rev Bras Ortop. 2011;46(1):14-17.

10. Hinterholz EL, Muhlen CA. Osteoartrose. Rev Bras Med. 2003;60:87-8.

11. Paula E, Leomil J, Oide MI, Mattar R Junior, Kimura LK, Okane seguimento. No grupo submetido à desnervação, a melhora da dor foi significativa em $44 \%$ dos casos e a melhora funcional de $42 \%$. O índice de satisfação foi de $87 \%$ no grupo da artrodese, enquanto no grupo da desnervação esse índice foi de $78 \%$. Contudo, essa diferença não foi estatisticamente significativa.

Apesar dos dados expostos, por tratar-se de uma técnica relativamente moderna, a desnervação do punho ainda carece de avaliação de resultados em grupos maiores e com seguimento prolongado, fazendo que ainda existam discussões sobre sua indicação como técnica isolada ou a necessidade de sua associação a técnicas tradicionais.

\section{CONCLUSÃO}

Conclui-se, portanto, que a desenervação parcial do punho (neurectomia do nervo interósseo) mostrou-se como uma boa opção para o controle álgico de pacientes com artrose no punho. Com possibilidade de postergar, ou até mesmo, evitar a artrodese total do punho. Devendo ser considerada como forma de tratamento para os casos avançados de artrose do punho antes de ser considerada a possibilidade de artrodese total do punho. Outros estudos semelhantes são necessários para reafirma os resultados aqui obtidos, uma vez que, são poucos os casos encontrados na literatura.

SY, et al. Ressecçäo do escafóide associada à "artrose dos quatros cantos" no tratamento de artrose da borda radial do carpo: estudos preliminares de cinco anos. Rev Bras Ortop. 1998;33(8):655-8.

12. Gomes EA, Armanelli F, Saliba GA. Pseudoartrose do tubérculo do escafoide em esqueleto imaturo: relato de caso. Rev Bras Ortop. 2012;47(4):513-16.

13. Nakamoto JC, Saito M, Cunha AP, Luques IU. Fratura do escafoide por estresse em ginasta: relato de caso. Rev Bras Ortop. 2009;44(6):533-55.

14 Franco LR, Simão LS, Pires EO, Guimarães EA. Influência da idade e da obesidade no diagnóstico sugestivo de artrose de joelho. ConScientiae Saúde. 2009;8(1):41-6.

15. Liposcki DB, Rosa F Neto. Prevalência de artrose, quedas e a relação com o equilíbrio dos idosos. Ter man. 2008;6(26):235-8.

16. Silva BA, Souza MV, Carrasco FM, Melo GA, Barreiros LE, Labronici PJ. Artrodese ainda é uma boa indicação na artrose não inflamatória do punho? Rev Bras Ortop. 2015;50(5):578-85.

17. Pardini AG Júnior, Gonçalves RF, Freitas AD, Chaves AB. Artrodese do punho com fixação mínima-preservando as articulações carpometacarpianas. Rev Bras Ortop. 2010;45(1):67-71.

18. Silva JB, Del Rio JT, Fernandes HF, Diniz S, Fridman M. Carpectomia proximal do carpo na artrose radiescafolunar. Rev Bras Ortop. 1997;32(11):894-8.

19. Godinho DR, Barros F, Barros A, Argotte W. Carpectomia proximal. Rev Bras Ortop. 1996;31(3):225-30.

20. França EN Bisneto, Freitas MC, Paula EJ, Mattar R Júnior, 
Zumiotti AV. Comparison between proximal row carpectomy and four-corner fusion for treating osteoarthrosis following carpal trauma: a prospective randomized study. Clinics. 2011;66(1):51-5.

21. Caetano EB, Morad JF, Camargo AA Neto, Franco RM, Santalla TP, Morad JF Filho. Contribuição ao estudo anatômico da inervação sensitiva do punho. Rev Fac Ciênc Méd Sorocaba. 2009;11(4):10-14.

22. Geldmacher J, Legal HR, Brug E. Results of denervation of the wrist and wrist joint by wilhelm's method. Hand. 1972;4(1):57-9.

23. Buck-Gramcko D. Denervation of the wrist joint. J Hand Surg Am. 1977;2(1):54-61.

24. Radu CA, Schachner M, Tränkle M, Germann G, Sauerbier M. Functional results after wrist denervation. Handchir Mikrochir Plast Chir. 2010;42(5):279-86. [in German].

25. Braga-Silva J, Román JA, Padoin AV. Wrist denervation for painful conditions of the wrist. J Hand Surg Am. 2011;36(6):961-6.
26. Storey PA, Lindau T, Jansen V, Woodbridge S, Bainbridge LC, Burke FD. Wrist denervation in isolation: a prospective outcome study with patient selection by wrist blockade. Hand Surgery. 2011;16(3):251-7.

27. Dellon AL, Mackinnon SE, Daneshvar A. Terminal branch of anterior interosseous nerve as source of wrist pain. J Hand Surg. 1984;9:316-22.

28. Weinstein LP, Berger RA. Analgesic benefit, functional outcome, and patient satisfaction after partial wrist denervation. J Hand Surg Am. 2002;27(5):833-9.

29. Riches PL, Elherik FK, Breusch SJ. Functional and patientreported outcome of partial wrist denervation versus the Mannerfelt wrist arthrodesis in the rheumatoid wrist. Arch Orthop Trauma Surg. 2014;134(7):1037-44.

\section{Como citar:}

Moreira JM, Viana ML, Facó YM, Lemos LQ, Bezerra GF, Cavalcante ML. Neurectomia do nervo interósseo anterior e posterior para controle da dor de pacientes com artrose de punho. Rev Med UFC. 2018 jul-set;58(3):59-64. 